\title{
Augsburg in Heidelberg - Der reformierte Umgang mit der Confessio Augustana
}

\section{Einführung}

In der Frage, wie die Reformierten sich zur Augustana verhalten haben, hat sich die Forschung hauptsächlich auf Calvin konzentriert. ${ }^{1}$ Der Genfer Reformator hatte schon seine Kritik an diesem Bekenntnis, aber hat sich doch immer hinter die Augustana gestellt und hatte keine Probleme, mit ihr übereinzustimmen. Grund dafür war auch, dass er die Unterschiede zwischen der Augustana und der reformierten Theologie zu gering achtete, um die Einheit der Kirche zu gefährden. Er unterzeichnete die Variata und bezeugte auch seine Übereinstimmung mit der Invariata, obwohl er dessen Artikel über das Abendmahl ablehnte. ${ }^{2}$ Die Einheit der Lutheraner und Calvinisten war auch ein Hauptthema in der Pfalz, wo von 1559 bis 1576 und von 1583 bis 1618 reformierte Fürsten regierten und die Heidelberger Universität eine internationale Hochburg calvinistischer Theologie war.

Der Religionsfriede, der 1555 in Augsburg geschlossen wurde, bedeutete für die Protestanten, dass sie nicht länger als Ketzer betrachtet wurden, das heißt, insofern sie zu denjenigen gehören, die das Augsburger Bekenntnis von 1530 unterschrieben hatten. In Artikel 5 wird festgelegt, dass jeder, der sich nicht entweder zur alten Kirche oder zu derjenigen der Augustana rechnet, von diesem Frieden ausgeschlossen ist, mit allen damit verbundenen Konsequenzen. Diese Bestimmung macht die Position der Reformierten im Deutschen Reich sowohl unklar als auch unsicher. Aus diesem Grunde verfolgten die Fürsten namentlich in der Pfalz die politische Absicht, eine Erweiterung der Bedingungen zu erreichen, so dass auch die Reformierten sich die Freiheit und den Schutz sichern

1 Danielle Fischer, Calvin et la Confession d'Augsburg, in: Wilhelm Heinrich Neuser (Hg.), Calvinus Ecclesiae Genevensis Custos: Die Referate des Congrès International des Recherches Calviniennes/International Congress on Calvin Research/Internationalen Kongresses für Calvinforschung vom 6. bis 9. September 1982 in Genf, Frankfurt a.M. 1984, 245-271; Willem Nijenhuis, Calvin and the Augsburg Confession, in: Ecclesia Reformata: Studies on the Reformation 1 (1972), 97-114; Jan Rohls, Die Confessio Augustana in den reformierten Kirchen Deutschlands, in: Zeitschrift für Theologie und Kirche 104,2 (2007), 207-245. Jan Rohls hat das Verhältnis der reformierten Kirchen in Deutschlands von Calvin bis Barth beschrieben und sich dabei, was das 16. und 17. Jahrhundert anbelangt, vor allem auf die kirchenpolitische Auseinandersetzung konzentriert.

2 Wim Janse, Calvin's Doctrine of the Lord's Supper, in: Perichoresis 10,2 (2012), 137-163.

Ә OpenAccess. () 2022 Herman J. Selderhuis, publiziert von De Gruyter. (cc) BY-NC-ND Dieses Werk ist lizenziert unter einer Creative Commons Namensnennung - Nicht kommerziell - Keine Bearbeitung 4.0 International Lizenz. https://doi.org/10.1515/9783110683868-014 
konnten. Von Seiten der calvinistischen Theologen versuchte man kräftig, den Lutheranern klar zu machen, dass auch die Calvinisten theologisch zu Luthers Nachfolgern gehörten, ja eigentlich die besseren Lutheraner waren. Dazu gehörte auch, dass immer wieder betont wurde, dass die Reformierten mit der Confessio Augustana übereinstimmten. Von lutherischer Seite wurden sie aber beschuldigt, dass sie in Wirklichkeit keinen einzelnen Artikel des Glaubensbekenntnisses in der rechten Weise glaubten. ${ }^{3}$

Um die vielen Diskussionen, die sowohl von der Kanzel aus als auch in Schriften in der Pfalz geführt wurden, zu beenden, erließ Johann Casimir im Jahre 1584 ein Mandat gegen diese Polemik. Die gegenseitige Lästerung und Verurteilung müsse unbedingt ein Ende finden. ${ }^{4}$ In diesem Mandat befahl Johann Casimir unter anderem, niemanden mehr lutherisch oder calvinistisch zu nennen, denn „wir sind alle eins in Christus, in dessen Namen wir getauft und erlöst werden.“5

Eine zentrale Rolle in dieser Diskussion spielt die Heidelberger Universität. Von deren Professoren wurden immer wieder Versuche unternommen, die Brücke zu den Lutheranern zu schlagen. Vertreter dieser sogenannten irenische Theologie oder Pfälzer Irenik waren vor allem David Pareus und Franciscus Junius. ${ }^{6}$ Diese Irenik stimulierte auch außerhalb der Pfalz, nach Einigungen zu suchen. Im Jahre 1628 publizierte der ungarische Superintendent János Samarjai ein Buch, in dem er aufzuzeigen versuchte, dass die Helvetische Konfession und die Confessio Augustana im völligen Einklang miteinander seien, und für seine Darstellung berief sich Samarjai wiederholt auf Abschnitte aus dem Irenicum des Pareus. ${ }^{7}$

Die Diskussion hielt trotzdem an. Während des Regensburger Reichstags 1594 zum Beispiel erschien ein anonymes Dokument, in dem behauptet wird, dass die

3 Hierzu: Herman J. Selderhuis, Luther totus noster est. The reception of Luther's thought at the Heidelberg theological faculty 1583-1622, in: Athina Lexutt/Volker Mantey/Volkmar Ortmann (Hg.), Reformation und Mönchtum, Tübingen 2008, 173-188.

4 „Pfaltzgraf Johann Casimirs / etc. Vormunds/ unnd der Churfürstlichen Pfaltz Administrators/ etc. Mandat und Befelch daß bey etlichen Kirchen und Schuln in der Chur Pfaltz/ eingerissene Condemnirn und Lestern uff der Cantzel und in den Schuln/ fürther zu underlassen und abzuschaffen,“ Heidelberg 1584.

5 Pfaltzgraff Johann Casimirs [...] Mandat und Befelch, daß bey etlichen Kirchen un[d] Schuln in der Chur Pfaltz eingerissene condemnirn und Lestern uff der Cantzel und in den Schuln, fürther zu underlassen und abzuschaffen, Heydelberg 1584, Aiiii.

6 Herman Johan Selderhuis, Frieden aus Heidelberg. Pfälzer Irenik und melanchthonische Theologie bei den Heidelberger Theologen David Pareus (1548-1622) und Franciscus Junius (1545-1602), in: Günter Frank/ Stephan Meier-Oeser (Hg.), Konfrontation und Dialog. Philipp Melanchthons Beitrag zu einer ökumenischen Hermeneutik, Leipzig 2006, 235-257.

7 Samarjai János/Bernhard Máte/Lippay György, Magyar Harmónia; Az Az, Augustana Es Az Helvetica Confessio Articulussinac Eggyező Értelme, Nyomtattatot Papan 1628. 
in Heidelberg gelehrte Theologie nicht mit dem Wort Gottes und dem Augsburger Bekenntnis übereinstimmt. Auf diese Weise wurde ein weiterer Versuch unternommen, die reformierte Pfalz außerhalb der Schutzvorkehrungen für den Augsburger Religionsfrieden zu halten. Auch anonym erscheint sofort eine Reaktion von Heidelberger Seite, wobei der Verfasser aber von sich sagt, dass dieses Werk geschrieben wurde, „durch einen Theologum der Augspurgischen Confession zugethan“ und dass seine Arbeit mit Approbation der Heidelberger theologischen Fakultät geschah. ${ }^{8}$

Interessant ist aber hier $\mathrm{zu}$ sehen, wie von drei Heidelberger Theologen in verschiedenen Perioden der Geschichte der Pfälzer Universität versucht wurde, die Einigung durch eine Kontextualisierung der Augustana zu erreichen.

\section{Zacharias Ursinus}

Der 1534 in Breslau geborene Ursinus ${ }^{9}$ ist vor allem bekannt geworden als Hauptverfasser des Heidelberger Katechismus. Sieben Jahre lang war er in Wittenberg Schüler von Philipp Melanchthon, was sich zeigt in dem von allen reformierten Kirchen als Bekenntnisschrift angenommen Katechismus ${ }^{10}$ sowie in seinen anderen Werken. Als leitender Theologe der Pfalz musste er sich mit der Augustana auseinandersetzten und tat dies in verschiedenen Veröffentlichungen. Beispielhaft für seine Beurteilung ist das Buch, das er 1564 publizierte, unter dem Titel Gründtlicher Bericht vom heiligen Abendmahl vnsers Herren Jesu Christi aus einhelliger Lere der heiligen Schrifft der alten rechtgläubigen Christlichen Kirchen und auch der Augsprugischen Confession. Gestellt durch der Uniuersitet Heydelberg Theologen. ${ }^{11}$

Diese Schrift wurde ab der Ausgabe 1566 erweitert mit Herrn Philippi Melanthonis Bedencken vber der spaltung vom Abendmal. Das Werk erschien in meh-

\footnotetext{
8 „Gegenbeweisung// Daß die Heidelbergische Theologen Got=//tes wort / der Augspurgischen Confession/ deroselben// Apologia/ und der Concordia Anno 36/ mit nichten unge=//meß lehren/ noch von ihrem Catechismo und vorigen // Schrifften im geringsten abweichen/ oder // wider einander seyn/ [...],“ Heidelberg 1594; Mehr Informationen dazu: Bibliotheca Palatina: Katalog zur Ausstellung vom. 8. Juli bis 2. November 1986 Heiliggeistkirche Heidelberg, hg.v. Elmar Mittler, Heidelberg 1986, Textband: 164-165.

9 Derk Visser, Zacharias Ursinus: the Reluctant Reformer: His Life and Times, New York 1983; Boris Wagner-Peterson, Doctrina Schola Vitae: Zacharias Ursinus (1534-1583) als Schriftausleger, Göttingen 2013 (Refo500 Academic Studies 13).
}

10 Derk Visser, Zacharias Ursinus (1534-1583). Melanchthons Geist im Heidelberger Katechismus, in: Heinz Scheible (Hg.), Melanchton in seinen Schülern, Wiesbaden 1997, 373-390.

11 Heidelberg 1564. 
reren deutschen und lateinischen Ausgabe und wurde 1566 auch in einer französischen und ein Jahr später in Emden in einer niederländischen Übersetzung ${ }^{12}$ veröffentlicht. ${ }^{13}$

Dieses Werk passt zu dem Bemühen, der reformierten Pfalz zu beweisen, dass auch die reformierte Tradition als rechtmäßig protestantisch anzuerkennen sei und deshalb unter den Schutz des Augsburger Religionsfriedens falle. ${ }^{14}$ Man kann deshalb auch die positive Bewertung der Augustana als kirchenpolitisch motiviert beurteilen, aber auch wenn es kirchenpolitisch oder sogar politisch war, muss es doch jedenfalls von reformierter Seite als inhaltlich begründet gelten und ist außerdem eine Motivation, die es wert ist, es als ökumenisch relevant näher zu betrachten. Es geht mir jetzt also nicht um die Frage, wie obengenannte Bemühungen zu bewerten sind und ob die Reformierten sich zurecht auf die Augustana berufen haben, aber wichtig ist, wie in diesem Fall Ursinus die Augustana als in einem bestimmten historischen Kontext entstandene Bekenntnisschrift beurteilt hat.

Der Gründtlicher Bericht erschien im Rahmen des Religionsgesprächs, das im April 1564 in Maulbronn zwischen Lutheranern aus Württemberg und Calvinisten aus der Pfalz geführt wurde, aber ohne die erwünsche Einigung endete. ${ }^{15}$ Ursinus verteidigt die reformierte Sakramentslehre zuerst mit Argumenten aus der Heiligen Schrift und an zweiter Stelle mit Argumenten aus der kirchlichen Tradition. Dann erst nimmt er die CA dazu und zwar die Invariata und versucht dann zu

12 Een claer bewys van het heylighe Avontmael onses Heeren Jesu Christi wt de eendrachtighe leere der H. Schriftueren der ouder rechtgelooven der Christelicker Kercken, en oock der Confessien van Augsburg. Door de Doctoren der H. Schrift der Universiteyt van Hydelberch. Wt de Hoochduytsche in Nederlandscher sprake ghetrouwelijcken overgheset. 1 Thess. 5. Proeft alle dinck en behoudt dat goedt is.

13 Eine Übersicht aller Editionen: Wagner-Peterson, Doctrina, 360 - 361.

14 Irene Dingel, Concordia controversa. Die öffentlichen Diskussionen um das lutherische Konkordienwerk am Ende des 16. Jahrhunderts, Gütersloh 1996 (Quellen und Forschungen zur Reformationsgeschichte 63); Christoph Strohm, ,Deutsch-reformierte‘ Theologie? Die kurpfälzische Reformation im Rahmen der frühneuzeitlichen Konfessionalisierung, in: Christoph Strohm/ Jan Stievermann (Hg.), Profil und Wirkung des Heidelberger Katechismus. Neue Forschungsbeiträge anlässlich des 450jährigen Jubiläums = The Heidelberger Catechism: Origins, Characteristics, and Influences. Essays in Reappraisal on the Occasion of its 450th Anniversary, Gütersloh 2015 (Schriften des Vereins für Reformationsgeschichte 215), 113-135.

15 Irene Dingel, Das Gespräch zu Maulbronn - Einigungsversuch zwischen dem lutherischen Württemberg und der reformierten Pfalz, in: Mittler, Bibliotheca Palatina, Textband: 160 -161; Volker Leppin, Das Maulbronner Religionsgespräch zwischen württembergischen und pfälzischen Theologen 1564, in: Irene Dingel/Volker Leppin/Kathrin Paasch (Hg.), Zwischen theologischem Dissens und politischer Duldung. Religionsgespräche in der Frühen Neuzeit, Göttingen 2018 (VIEG. Beih. 121), 161-182. 
zeigen, dass die Invariata und der Heidelberger Katechismus grundsätzlich übereinstimmen oder auf jedem Fall sich nicht widersprechen, wenn es um die Abendmahlslehre geht.

Nach seiner Auffassung wird zu Unrecht davon ausgegangen, dass die CA die Meinung vertritt, dass Christus in den Zeichen von Brot und Wein leiblich präsent ist. Artikel 10 lehrt zwar die Gegenwart Christi in den Elementen, aber nicht eine physische Gegenwart. Das zweite Missverständnis ist die Meinung, dass die CA davon ausgehe, dass auch Ungläubige durch Brot und Wein am Leib und Blut Christi teilhaben können. Deutlich aber betont das Augsburger Bekenntnis in Artikel 13 die Notwendigkeit des Glaubens, um an der Erlösung Christi teilzuhaben. Dies zu bezeugen wäre überflüssig, wenn Christus dermaßen leiblich präsent sei, wie oft behauptet wird.

Basierend auf diesen und anderen Annahmen kommt Ursinus zu dem Schluss, dass das Augsburger Bekenntnis und auch seine Apologia von 1531 in keiner Weise im Widerspruch zur Lehre vom Sakrament steht, wie sie von den Reformierten erklärt wurde, sondern diese vielmehr bestätigt. Umgekehrt lehren die Reformierten nichts über das Sakrament Christi, das dem Augsburger Bekenntnis von 1530 oder der Heiligen Schrift widerspricht. ${ }^{16}$

Es ist klar, dass die Argumentation von Ursinus eine kirchenpolitische Komponente hat. Sein Kurfürst Friedrich III. musste sich am Augsburger Reichstag 1566 für seine reformierte Position verteidigen und brauchte theologische Argumente, um zu beweisen, dass seine Position genuin protestantisch und also nicht gesetzwidrig war. ${ }^{17}$ Deshalb hat er immer wieder bezeugt, dass er sich dem Augsburger Bekenntnis von 1530 voll und ganz angeschlossen hat. Trotzdem ist auch aus den anderen Werken von Ursinus klar, dass diese Auffassung zu seiner theologischen Grundidee gehört.

Für unser Thema ist aber wichtig, dass er die Augustana kontextualisiert. ${ }^{18} \mathrm{Er}$ versteht auch, dass an der Augustana Kritik geübt werden kann und auch soll, aber er weist darauf hin, dass die Augustana in einer bestimmten historischen Situation entstanden ist. Die Finsternis des Papsttums war dermaßen groß, dass es kein Wunder ist, dass nicht ,alle stück der Lehre/ gerad auff einmal so hell und

16 Ursinus, Gründtlicher Bericht, 365.

17 Walter Hollweg, Der Augsburger Reichstag von 1566 und seine Bedeutung für die Entwicklung der reformierten Kirche und ihrer Konfessionen, Neukirchen 1964.

18 Wolgast spricht von einer „[...] Autoritätsrelativierung durch die Historisierung des Textes.“, Eike Wolgast, Die Heidelberger Irenik und die Praxis der Toleranz in der Kurpfalz (1559-1622), in: Herman Selderhuis/J. Marius J. Lange van Ravenswaay (Hg.), Reformed Majorities in Early Modern Europe, Göttingen 2015 (RFO 500 Academic Studies 23), 181-201, 187. 
lauter haben mögen an tag bracht und erkläret werden“. ${ }^{19}$ Zweitens haben die Evangelischen versucht, in ihren Formulierungen vorsichtig zu sein, um Frieden und Einheit der Kirche zu bewahren. Das ist der Grund, dass sie „die Bäptischen Irrthume unnd Mißbrauch auffs aller glimpffichste/ undd lindeste so möglich anzugreiffen sich beflissen haben.“ Um klar zu machen, dass dies nicht eine Ausrede von Ursinus ist, weist er darauf hin, dass auch in den Vorreden zur $\mathrm{Au}$ gustana sowie zur Apologia zu lesen ist, dass man versucht hat, Einigkeit zu bewahren. Die Augustana steht also in einer Entwicklung, in der die rechte Lehre immer deutlicher hervortrat. Für Ursinus gibt es also nicht nur eine inhaltliche Übereinstimmung zwischen der Abendmahlslehre der Augustana und derjenigen der Reformierten, sondern man muss auch verstehen, in welcher Phase der Reformation und mit welchem Ziel diese verfasst wurde. Ursinus hat diese Kontextualisierung der Augustana auch später stets betont wie zum Beispiel in der Reaktion auf das Konkordienbuch, das er 1581 im Namen der Pfälzischen Theologen verfasste und das als Admonitio Christiana bekannt ist. ${ }^{20}$ Dort sagt Ursinus, dass die Augustana nur eine Konfession unter anderen sei und sie deshalb nicht verabsolutiert werden soll. ${ }^{21}$

\section{Georg Sohn (1551-1589)}

Georg Sohn gilt als unverblümter Anhänger von Melanchthon. ${ }^{22}$ Geboren 1551 in Roßbach in der Wetterau, studierte er Theologie in Wittenberg und in Marburg. An dieser hessischen Universität wurde er 1574, d. h. im Alter von 23 Jahren, Professor

19 Ursinus, Gründtlicher Bericht, 363.

20 Zacharias Ursinus, De Libro Concordiae quem vocant, A quibusdam Theologis, nomine quorundam Ordinum Augustanae Confessionis, edito, Admonitio Christiana: Scripta à Theologis et Ministris Ecclesiarum in ditione Illustrißimi Principis Iohannis Casimiri Palatini ad Rhenum Bavariae Ducis, \&c., Neustadii in Palatinatu 1581. Eine ausführliche Beschreibung des Inhalts sowie des historischen Kontextes gibt: Irene Dingel, Concordia controversa. Die öffentlichen Diskussionen um das lutherische Konkordienwerk am Ende des 16. Jahrhunderts, Gütersloh 1996 (Quellen und Forschungen zur Reformationsgeschichte 63), 141-148.

21 Irene Dingel qualifiziert diese Position als „eine deutliche Umwertung“ der Augustana. Dingel, Concordia, 148.

22 ADB, Bd. 34, 543-544; Dagmar Drüll, Heidelberger Gelehrtenlexikon 1386-1651, Berlin/ Heidelberg 2002, 507-508; Theodor Mahlmann, Theologie, in: Barbara Bauer (Hg.), Melanchthon und die Marburger Professoren, Bd. 2, Marburg 1999, 628-645; Nam Kyu Lee, Die Prädestinationslehre der Heidelberger Theologen 1583-1622. Georg Sohn (1551-1589), Herman Rennecherus (1550-?), Jacob Kimedoncius (1554-1596), Daniel Tossanus (1541-1602), Göttingen 2009 (Reformed Historical Theology 10). 
für Theologie. Mit seinem Kollegen dort, Aegidius Hunnius, entstand ein Konflikt, in dem deutlich wurde, wie sehr Sohn ein Schüler von Melanchthon ist. Da der lutherische Hunnius jedoch die Unterstützung des Marburger Landgrafen Ludwig IV. genoss, nahm Sohn 1584 eine Anstellung in Heidelbergs an, wo er bis zu seinem frühen Tod 1589 wirkte. 1588 erschien dort Sohns wichtigstes Werk, nämlich seine Synopsis Corporis Doctrinae Philippi Melanchthonis. ${ }^{23}$

Wenn Georg Sohn sich entscheidet, Vorträge über das Augsburger Glaubensbekenntnis zu halten ${ }^{24}$ - die erst 1609, also zwanzig Jahre nach seinem Tod publiziert wurden -, weist er selbst auf den Zweck hin. Zunächst geht es um ein sehr wichtiges Bekenntnis der Evangelischen Kirchen in Deutschland, das die Theologiestudenten sehr gut kennen müssen, um die Inhalte der Augustana in Zukunft weitergeben zu können. Gleichzeitig geht dieses Bekenntnis aber auch auf alle Punkte ein, die heute wieder diskutiert werden. ${ }^{25}$ Aus dieser positiven Bewertung heraus macht er auf der Grundlage der Augustana die reformierte und nach Heidelberger Ansicht wirklich reformierte Meinung deutlich und weist gleichzeitig die Missverständnisse von Trient und dem Luthertum zurück. Deshalb beschäftigt sich Sohn nicht mit allen Artikeln und beschränkt sich auf wenige Artikel. Zunächst diskutiert er die Lehre Gottes und dies im Zusammenhang mit den Diskussionen über die Dreieinigkeit mit Muslimen und mit einigen protestantischen Gegnern dieser Lehre aus dem 16. Jahrhundert. ${ }^{26}$ Die Christologie wird wegen der Doktrin der Ubiquität Christi diskutiert. Die Vorlesungen über die Erbsünde berühren die Diskussion mit Rom und mit den Lehren von Flacius Illyricus, und seine Erwägungen zum Thema des freien Willens beziehen sich auf die Diskussion mit Rom sowie mit den Lutheranern. Wenn er sich schließlich mit der Rechtfertigung beschäftigt, kann er sich zunächst auf die Diskussion mit Rom konzentrieren, aber sobald er die Rechtfertigungslehre mit der Prädestinationslehre verbindet, geht es vor allem um die Polemik mit den Lutheranern. Die Themen, mit denen sich Sohn hier beschäftigt, sind vor allem diejenigen, die auch in der Heidelberger Polemik ständig behandelt werden.

23 Synopsis Corporis Doctrinae Philippi Melanchthonis Thesibus beviter comprehensa et anno 1582. Marpurgi in privata schola ad disputandum proposita, et nunc primum edita a Georgio Sohn Sacrae Theologiae Doctore et Professore, Heidelberg 1588. Hier zitiert nach der Ausgabe der Gesammelten Disputationen von Sohn: Theses de plerisque locis theologicis, in Academiis Marpurgensi et Heidelbergensi ad disputandum propositae, Herborn 1609.

24 Diese Vorlesung wurde 1609 durch seinen Sohn herausgegeben als Teil 2 von Sohns gesammelten Werken, Tomus secundus: continens exegesin praecipuorum articulorum Augustanae Confessionis, Heidelberg 1609 und hat einen Umfang von mehr als 100 Seiten.

25 Tractat haec Confessio praecipuos Locos communes Theologiae, \& praecipuas controversias Ecclesiasticas praesertim huius temporis attingit. Sohn, Exegesin, 4.

26 Sohn, Exegesin, 85-188. 
In seinen Vorträgen zur Augsburger Konfession gibt Sohn einen dogmatischhistorischen Überblick über die Auseinandersetzung um die Sakramente aus der Sicht der Diskussion über die Allgegenwart. Er glaubt, dass eine solche sachliche Übersicht notwendig ist, weil reformierte Menschen und Lutheraner sich missverstehen, sich manchmal fälschlicherweise gegenseitig beschuldigen, und dass sie nicht immer wissen, was die Position des anderen ist. ${ }^{27}$ Seiner Meinung nach entstand das Problem vor allem, als Luther in der Diskussion mit Zwingli den Begriff der Allgegenwart zu verwenden begann. ${ }^{28}$ Sohn versucht dann, aus Luthers Werken deutlich zu machen, dass Luther hiermit etwas ganz anderes bedeutete als die Lutheraner von heute. Auch Luther hat sich nachdrücklich von diesem Konzept distanziert ${ }^{29}$ und daher ist dieser Begriff weder in der Augustana noch in Luthers Spätwerk, noch bei Melanchthon oder in den Schmalkaldischen Artikeln zu finden. Der Begriff war vergraben, so sagt Sohn, bis Brenz ihn wieder ausgrub. ${ }^{30}$ Sohn geht dann auf einigen Seiten gegen Brenz und Jacob Andrea vor und erklärt, dass sie das, was Luther gesagt hat, um Dinge ergänzt haben, die sie als Luthers präsentieren, aber bei Luther nicht vorkommen. ${ }^{31}$ Sie zitieren auch aus Luthers Werken von 1527/28, einer Zeit, in der er heftig polemisierte. Gerade deshalb ist es nach Sohn falsch, aus solchen Schriften wesentliche Schlüsse zu ziehen, die nicht durch Zitate aus Luthers eher theoretischen Werken gestützt werden können. Nach diesem Überblick widmet Sohn viele Seiten dem Verständnis der Verbindung zwischen den beiden Naturen Christi. ${ }^{32}$

\section{Heinrich Alting (1583-1644)}

Alting, ${ }^{33}$ geboren in Emden, ausgebildet in Groningen und Herborn, war der Sohn des berühmten reformierten Pastors Menso Alting. Er arbeitete acht Jahre lang in

27 Nam plerique affectibus aut praejudiciis praeoccupati, sinistris interpretationibus, calumniis \& convitiis indulserunt. Sohn, Exegesin, 348.

28 [...] et Lutherus primus omnium Ubiquitatem corporis Christi contra adversarios suos duobus annis \& duobus scriptis illis asseruit, hoc est, semina Ubiquitatis in Ecclesia sparsit. Sohn, Exegesin, 349.

29 Lutherus [...] etiam doctrinam Ubiquitatis, cujus antea quaedam semina sparserat, expresse rejecit. Sohn, Exegesin, 355.

30 Sohn, Exegesin, 357.

31 [...] sed etiam novis additionibus, de quibus nihil apud Lutherum reperitur [...], Sohn, Exegesin, 367.

32 Sohn, Exegesin, 378-542.

33 Thomas Klöckner, Heinrich Alting (1583-1644). Lebensbild und Bedeutung für die reformierte Historiografie und Dogmengeschichtsschreibung des 17. Jahrhunderts, Göttingen 2019 
der Pfalz, so dass dies für Heinrich ein vertrautes Gebiet war. 1613 wurde Heinrich Alting zum Professor für Dogmatik (Professor locorum communium) in antiquissima \& tunc florentissima Heidelbergensi Academia ${ }^{34}$ ernannt, eine Position, die 1616 auf die des Leiters des Collegium Sapientiae erweitert wurde. 1618 sollte Alting den Vorsitz im Alten Testament erhalten, aber er überließ ihn Abraham Scultetus. Zusammen mit diesem und Paul Tossanus bildete Alting die pfälzische Delegation bei der Synode von Dordrecht. 1622 entkam Alting glücklich der Kriegsgewalt und ließ sich in Den Haag nieder, wo er Erzieher von Friedrich Heinrich, dem ältesten Sohn des Winterkönigs Frederik V., der in die Niederlande floh, wurde. 1627 nahm Alting den Ruf an die Universität Groningen an und lehrte dort bis zu seinem Tod 1644 Dogmatik. Hier erweiterte er die traditionelle dreifache Berufsteilung (theologia didactica, theologia elenchtica und problemata theoretica et practica) durch eine theologia historica, in der er sich mit der Entwicklung der Lehre beschäftigte und damit zu den Begründern der Geschichte der Dogmatik gehört. ${ }^{35}$

Heinrich Alting diskutiert in seiner Exegesis Logica et Theologica Augustanae Confessionis die Augsburger Konfession aus der Sicht, ob die deutschen reformierten Kirchen zu der Gruppe gehören, die sich um diese Konfession versammelt haben. ${ }^{36}$ Diese Schrift wurde nicht in die Ausgabe seiner Heidelberger Schriften

(Reformed Historical Theology 56); Martin Tielke (Hg.), Biographisches Lexikon für Ostfriesland, Bd. 1, Aurich 1993, 22-24; Biografisch Lexicon voor de Geschiedenis van het Nederlandse Protestantisme, hg.v. C. Houtman/J. van Sluis/J. H. van de Bank, Kampen 2001, Bd. 2, 22-24; Und die in diesem Lexikon erwähnte Literatur; Portrait und Biographie in: Effigies et Vitae Proessorum Groningae et Eomlandiae, Groningen 1654, 88-101.

34 Effigies et Vitae, 91.

35 Otto Ritschl, Dogmengeschichte des Protestantismus. Grundlagen und Grundzüge der theologischen Gedenken- und Lehrbildung in den protestantischen Kirchen, Bd. 1, Göttingen 1908, 77; 1660 veröffentlichte J. H. Hottinger eine dogmatische Übersicht mit explizitem Bezug auf die Methode des Alting: Cursus Theologicus; methodo altingiana, qua, non modo Definitiones eius proponuntur [...], Duisburgi ad Rhenum 1660.

36 Heinrich Alting, Exegesis logica et theologica Augustanae confessionis: cum appendice problematica: num ecclesiae reformatae in Germania pro Sociis Augustanae confessionis agnoscendae et habendae sint? Acc. syllabus controversiarum, quae reformatis hodie intercedunt cum Lutheranis, Amstelodami 1652; Vgl. zu dieser Problematik: Herman J. Selderhuis, Frieden aus Heidelberg: Pfälzer Irenik und melanchtonische Theologie bei den Heidelberger Theologen David Pareus (1548-1622) und Franciscus Junius (1545-1602), in: Günter Frank/ Stephan Meier-Oeser (Hg.), Konfrontation und Dialog: Philipp Melanchthons Beitrag zu einer ökumenischen Hermeneutik, Leipzig 2006 (Schriften der Europäischen Melanchtonakademie 1); und neuerdings: Christoph Strohm, Die Universität Heidelberg als Zentrum der späten Reformation, in: Ulrich A. Wien/Volker Leppin (Hg.), Kirche und Politik am Oberrhein im 16. Jahrhundert. Reformation und Macht im Südwesten des Reiches, Tübingen 2015 (Spätmittelalter, Humanismus, Reformation 89), 
aufgenommen und nach seinem Tod durch seinen Sohn Jakob veröffentlicht. In einer Heidelberger Disputation mit dem Titel Disputatio Harmonica Confessionum Augustanae, Palatinae, et Helveticae Orthodoxum Consensum exhibens aus dem Jahr 1620 vergleicht er die Confessio Augustana, den Heidelberger Katechismus und die Confessio Helvetica posterior miteinander. Sein Ergebnis ist, dass diese drei Bekenntnisschriften im Wesentlichen miteinander übereinstimmen, aber dass es in der Abendmahlslehre doch Unterschiede gibt. Diese Unterschiede betreffen aber nicht die Sache, sondern nur die Art und Weise, wie formuliert wird, und deshalb sind diese Unterschiede auch nicht kirchentrennend. Seine Schlussfolgerung ist, dass sie in allem, außer dem Sakrament gleich sind, aber Alting sagt, dass dies kein grundlegender Unterschied ist, weil es eine Übereinstimmung über das Wesen der Angelegenheit gibt. ${ }^{37}$ Auch die Reformierten bezeugen die Wirksamkeit des Sakraments und es sei lasterhaft, das zu verneinen. ${ }^{38}$ Die Schlussfolgerung von Alting, die nicht nur in dieser Debatte, sondern auch in seiner gesamten Arbeit zu diesem Thema zu hören ist, lautet, dass das deutschreformierte Volk auch zu den Verbündeten der Augustana gehört. Sie werden als reformiert bezeichnet, weil sie sich von Rom gelöst und die Lehre und Bräuche gemäß der Heiligen Schrift neu geordnet haben. Sie werden evangelisch genannt, weil sie begonnen haben, das Evangelium der Gnade wieder zu verkünden. Sie sind Protestanten, weil sie 1529 auch auf dem Reichstag in Speyer protestiert haben. ${ }^{39}$ Auch in dieser Hinsicht gehören sie zur Societas Augustane Confessionis. ${ }^{40}$

202-204; Sowie speziell zu Alting: Pierre-Olivier Léchot, Entre Irénisme et controverse. La réécriture historique de la différence confessionnelle chez le théologien paltin Heinrich Alting (15831644), in: Bertrand Forclaz (Hg.), L'expérience de la différence religieuse dans l'Europe moderne (XVIe-XVIIe siècles), Neuchâtel 2012, 356-363 (der Autor spricht im Falle Altings von „historiografischem Irenismus“, was noch einer genaueren Analyse bedarf; s. unten Kap. 3.3.3); Gustav A. Benrath, Reformierte Kirchengeschichtsschreibung an der Universität Heidelberg im 16. und 17. Jahrhundert, Speyer 1963 (Veröffentlichungen des Vereins für Pfälzische Kirchengeschichte 9). 37 Heinrich Alting, Exegesis logica; Vgl. J. F. G. Goeters, in: Joachim Staedtke/Gottfried W. Locher (Hg.), Glauben und Bekennen: 400 Jahre Confessio Helvetica Posterior, Zürich 1966, 92-94.

38 Calumnia igitur inanis est, quod Ecclesiae Reformatae negent efficaciam Sacramentorum; naturam eorum ac definitionem multis modis corrumpant; usum denique Sacramentorum in electis vocatis nullum relinquant. Contrarium enim dictae Confessiones demonstrant. Alting, Exegesis Logica, 1 (hier zit. die letzte konkludierende These der Thesenreihe LXVIII-LXXI).

39 Alting, Exegesis Logica, 64. Ecclesia Reformata vocantur, quae secessione facta a Papatu Romano, doctrinam ac ritus sacros reformarunt, [...]. Eadem Ecclesiae dici solent Euangelica: quia Euangelium aeternum, Apoc. 14. v. 6. quod Antichristus perpetuis tenebris oppressum cupivit, [...] propagarunt. Denique etiam Ecclesiae Protestantium appellantur: quia Magistratus earum una protestati sunt adversus iniquissimum decretum Comitiorum Spirensium Anno Christi 1529. quo proscribebantur; Alting, Exegesis Logica, 114-115. 
Für unser Thema noch wichtiger ist, dass Alting die CA in einen historischen Rahmen einordnet. Bevor er mit der Auslegung der CA beginnt, beschreibt er in seinen Prolegomena in Augustanam Confessionem ${ }^{41}$ die Entstehungsgeschichte dieser Schrift, nicht um dessen Inhalt, sondern um dessen Status zu relativieren. Damit man die CA besser verstehen kann, sind vier Aspekte zu beachten. Erstens wie die CA entstanden ist, zweitens wie sie bewahrt und erweitert wurde, drittens was der Inhalt ist und viertens was das Ziel der CA war. Er nennt die CA „eine besondere Konfession einiger Deutscher Kirchen“. Schon das „einiger Deutscher Kirchen“ ist bedeutend, aber mehr noch der Begriff particularis. Grund für diese Charakterisierung ist, dass die CA „nicht aufgestellt, approbiert und angeboten wurde durch die katholische Kirche (Ecclesia Catholica), sondern nur durch einige bestimmte Kirchen und deren Magistrate und Doctoren in Deutschland“. Es geht also um eine Deutsche Bekenntnisschrift und dann noch die Bekenntnisschrift einiger Deutschen Kirchen. Sie ist nicht aus der Catholica entstanden und hat, wie Alting sagt, deshalb auch keine höhere Autorität als andere Konfessionen, die zur gleichen Zeit oder danach entstanden sind. ${ }^{42}$ Deshalb sind auch nicht alle Kirche an die CA gebunden und sie ist auch nicht norma \& regula fidei \& religiones ${ }^{43}$ wie die drei altkirchliche Symbole. Daraus folgt, dass man denjenigen, der davon abweicht, auch nicht als Ketzer schelten kann. Die CA ist die Konfession einiger Deutscher Kirchen sowie auch der Kirchen in Böhmen, Frankreich, England, der Schweiz und der Niederlande, die ihre eigenen Konfessionsschriften haben.

Was die Entstehung anbelangt, unterscheidet Alting zwischen den Fürsten deren Namen er auflistet - als diejenigen, die die CA präsentiert haben und deshalb die eigentlichen Produzenten sind, und den Theologen, die für die Inhalte verantwortlich sind. Auch listet Alting die Namen der Theologen auf, die an der CA gearbeitet haben, nennt aber ausdrücklich Luther als Hauptverfasser. Er hat die siebzehn zur Debatte stehende Artikel als Skizze beschrieben und Melanchthon hat das Ganze dann verfeinert. Alting weist darauf hin, dass es zwei Fassungen der CA gibt und dass in der ersten Fassung bezüglich des Herrenmahls eine Formulierung der Präsenz Christi gewählt wurde, die sich absichtlich nicht zu weit von der Transsubstantiationslehre entfernen wollte. In der zweiten Fas-

40 Alting, Exegesis Logica, 65.

41 Alting, Exegesis Logica, 1.

42 Ideoque nec plus authoritatis obtinere potest, quam aliarum Ecclesiarum particulares Confessiones: quae vel circa illa tempora, vel etiam aliquanto post confectae \& editae sunt. Alting, Exegesis Logica, 1.

43 Prolegomena, 4. 
sung aber, die 1542 - als Luther noch lebte und davon wusste ${ }^{44}$ - entstand, sind einige Artikel geändert und erweitert worden, und das gilt auch für den 10. Artikel zur Abendmahlslehre. Eine weitere Kontextualisierung der Augustana ergibt sich aus Altings Einführung, in der er sagt, die Augustana stand im Rahmen des kaiserlichen Ziels, eine Einigung zwischen Rom und den Protestanten zu erreichen. ${ }^{45}$

\section{Zum Schluss}

In dem frühneuzeitlichen Calvinismus setzte man sich mit dem Augsburger Glaubensbekenntnis auseinander auf Grund der Überzeugung, mit den Anhängern dieses Bekenntnisses auf gemeinsamer Basis zu stehen. Dazu stützten sich die Reformierten auf die CA-Interpretation Melanchthons und auf das Argument, dass die Invariata in ihrem Kontext noch stark mit den katholischen Gegnern rechnen musste. ${ }^{46}$ Die Überzeugung der gemeinsamen Grundlage zeigte sich auch darin, dass die reformierte Konfessionalisierung in Deutschland immer Hand in Hand mit der Anerkennung der CA ging, aber dann mit der Variata, die dann oft friedlich neben dem Heidelberger Katechismus existierte. ${ }^{47}$

44 [...] vivente Luthero\& sciente [...], Prolegomena, 2.

45 [...] nempe Pontificiorum \& Protestantium, conciliatione acturus [...], Prolegomena, 1.

46 Prolegomena, 231.

47 Jan Rohls, Die Confessio Augustana in den reformierten Kirchen Deutschlands, in: Zeitschrift für Theologie und Kirche 104, 2 (2007), 226-227. 